\title{
Design and Fabrication of a Mounting Device for Connecting a Motorcycle and a Sidecar
}

\author{
Engr. Juanito P. Maneclang Jr. ${ }^{1}$, Dr. Jeff Galapon Pereyras ${ }^{1 *}$ \\ ${ }^{1}$ Pangasinan State University - Lingayen Campus, Philippines \\ *jpereyras@psu.edu.ph
}

\begin{abstract}
The fabricated mounting device was a utility model whose function was to connect and attached a motorcycle to its sidecar in seconds. This study employed a developmental type of research. A mounting device for connecting a motorcycle to a sidecar, comprising: a post having a pair of bores mounted to a motorcycle chassis; a support bar having a pair of dowels attached to a reinforce bar of the said sidecar and fasten to the bores of the said post; a sleeve connector having an opposing side opening forming thereon an inside C-channel adapted to slide and interconnect said post and support bar; and a threaded locking bore provided on one side of said sleeve connector and fastening means screwably mounted to said threaded locking bore; wherein said means include a bolt threaded to said bore to secure the sleeve connector to said post and support bar. The demonstration, interview, and consultation with the experts, particularly the faculty members of the Mechanical Technology Department of Pangasinan State University - Lingayen Campus, and the end-users, a group of veteran tricycle users, the said utility model can be forceful and advantageous compared to the conventional way of connecting a motorcycle to its sidecar. The mounting device for connecting a motorcycle and a sidecar was patented under Industrial Design and Utility Model Categories by the Intellectual Property Office of the Philippines.
\end{abstract}

Key words: Motorcycle, Sidecar, Mounting Device, Utility Model.

\section{INTRODUCTION}

A motorcycle is a recreational vehicle with two (2) wheels. Motorcycle construction differs extensively for many purposes: long-distance driving, commuting, activities such as racing, and off-road biking. Despite reduced prices and better fuel efficiency, motorcycles are considered utilitarian in developing countries. The low fuel consumption of motorcycles has drawn concern from environmentalists in the Philippines because of low carbon emissions and those impacted by higher fuel prices. A sidecar is a one-wheeled automobile mounted to the side of a motorcycle, rendering it a three-wheeled bike. The motorcycle attached to a sidecar is most popularly called a tricycle in the Philippines.

Tricycles are local customs of the vehicle in the Philippines and are a shared means of community or private transport. Such public transport vehicles, like taxis, just ply the path you have planned or are for-.The tricycle is a famous means of transport in the cities and small municipalities. Tricycles are constructed in several different types and they vary from one locale to another and are typically rendered locally by constructing a sidecar and attaching it to a motorbike. Motorcycles and sidecars are typically enclosed, but not always with the same canopy.

According to Wallace F. Mitchell, US Patent 4,385,770, for the most part, motorcycle sidecars used a single wheel dialled around a fixed horizontal axis that is typically aligned with the corresponding motorcycle rear axle. Those sidecar frames are rigidly attached to related motorcycle frames. The usual manner by which a sidecar is connected to a motorcycle is by a fastener made of metal plates with bolts and knots. The time consumed in connecting a sidecar to a motorcycle is a hassle as well as in the disassembly [1].

To solve the problems being encountered by the tricycle drivers, the authors designed and fabricated a utility model that relates generally to vehicle connecting devices and more particularly to a mounting device for motorcycle and sidecar to hasten the assembly and dismantling of the sidecar to and from the motorcycle.

\section{LITERATURE REVIEW}

Basic instructions on installing the sidecar to the motorcycle. It is necessary to position the two upper mounts on the motorcycle frame as high as probable and as far apart as possible. It is safest to sit next to the mainframe junction or the secured area on the wall. The lower two mounts on the wheel, too, will be as far away and as short as possible. Decent triangulation is vital to strengthening. There is a need to distribute the load across a subframe on some motorcycles, and to add it to as many locations as probable. The arrangement of the mounts to cross to the other side of the frame is a reasonable approach of providing the rig with more lateral power. It is one field that is frequently ignored. A sidecar contributes a ton of side load to the motorcycle and that must be taken into consideration in every mounting situation. The sidecar should be adjusted and the set-up level using a certain tool called "bubble level" before connecting the sidecar to the motorbike. The motorcycle rider is supposed to sit back on the motorbike compressing the height suspension. When the rider stays on the wheel, the second individual will use tiedowns to keep the rigid suspension in place as the rider gets off. When rode out on the lane, it would entail the lowering of the vehicle. Fixed the mounts on it. Then it is set to place the sidecar 
next to the motorcycle, then attach the two sides of the bike to the side of the sidecar. Wheel lead will be calculated as similar to $10 \%-15 \%$ of the motorcycle wheelbase estimated from the front to the rear axles as possible. It can now snug the mounting bolts with wheel lead and toe-in set and Test the set up again, and the bolts are stretched. Find out the lean on the wheel, then add it to the top two struts. A few times this might be appropriate to change the lean out and allow the rig to go straight down the road without moving on one side. Tire pressure on the sidecar tire is also tested. Lower pressure on the tire may give your passenger a more comfortable ride. Many sidecar frames may have lower mounting positions which are non-adjustable. They should be broken off, so sidecars can have different fittings to make it simpler to change and position the sidecar securely. Sidecars are common in creating a minor head movement at lower speeds at the handlebars. This can also be reduced to a minimal by carefully adjusting the toe-in at $1 / 8$ "at a time. Other ways of minimizing track to reduce steering strength are possible and flat surface tires are often available in small sizes. However, if the steering is modified, the rig may become a dedicated three-wheeled vehicle and cannot be comfortably driven as a two-wheeled car [2].

Tricycles are one of the most convenient ways to get around, particularly when walking too far is too close to bother bringing a car. Going a few blocks away to the desired destination, riding a motorcycle regularly is one of its advantages. It's more convenient to ride a tricycle than driving a car along a short, daily route. Apart from parking constraints, it is such a troublefree means to get to the desired destination. Another advantage of taking a tricycle is during the morning rush and other peak hours of commuters, it adds convenience on the part of the commuters[3].

Riding on tricycles gets commuters faster to their main destination and takes commuters directly to their houses, unlike buses that drop on the junction or unloading area. Another tricycle helps the commuter to locate street addresses that a stranger does not know. This tricycle can easily be gotten as they are all everywhere. They are a safer means of transport compared to motor-bike. Tricycles are almost like conventional cars with a roof that protects the commuter from the sun and rain. Riders and commuters do not have to buy a helmet to drive a tricycle [4].

\section{OBJECTIVE OF THE STUDY}

The primary aim of this developmental research was to design and developed a mounting device for connecting the motorcycle and its sidecar and hasten the assembly and dismantling of a motorcycle and a sidecar. This study was intended to address the problems being encountered by the tricycle users particularly in assembling and dismantling of the sidecar to and from the motorcycle.

\section{MATERIALS AND METHODOLOGY}

\subsection{Materials}

Referring to Figure 1, there is shown the present utility model for a mounting device for motorcycle and sidecar designated as
60. The mounting device 60 comprising connector assembly 10 is mounted to the post 20 and support bar 30 as shown in Figure 2.

Referring particularly to Figure 2, the post 20 is made of the flat bar provided with a pair of bores 21 which is rigidly fastened to the motorcycle chassis 40 . The support bar 30 is made of the flat bar provided with dowels 31 configured to be mounted to the said post 20 . The support bar 30 is rigidly connected to the reinforced bar arm 50 of a sidecar.

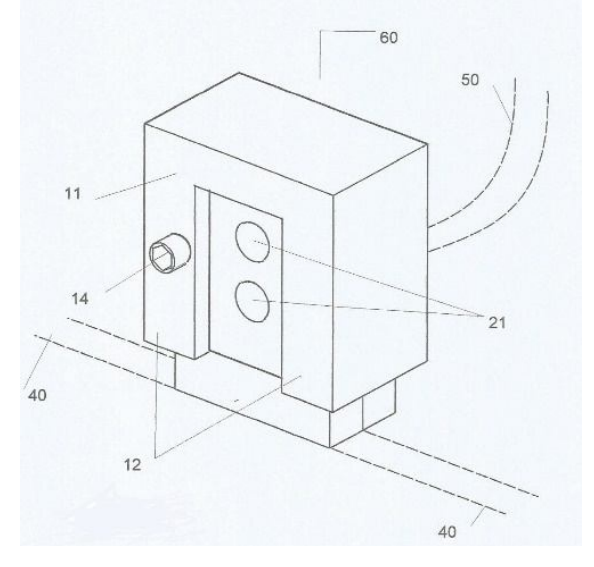

Figure 1: Perspective View of the utility model, which shows the design and its parts
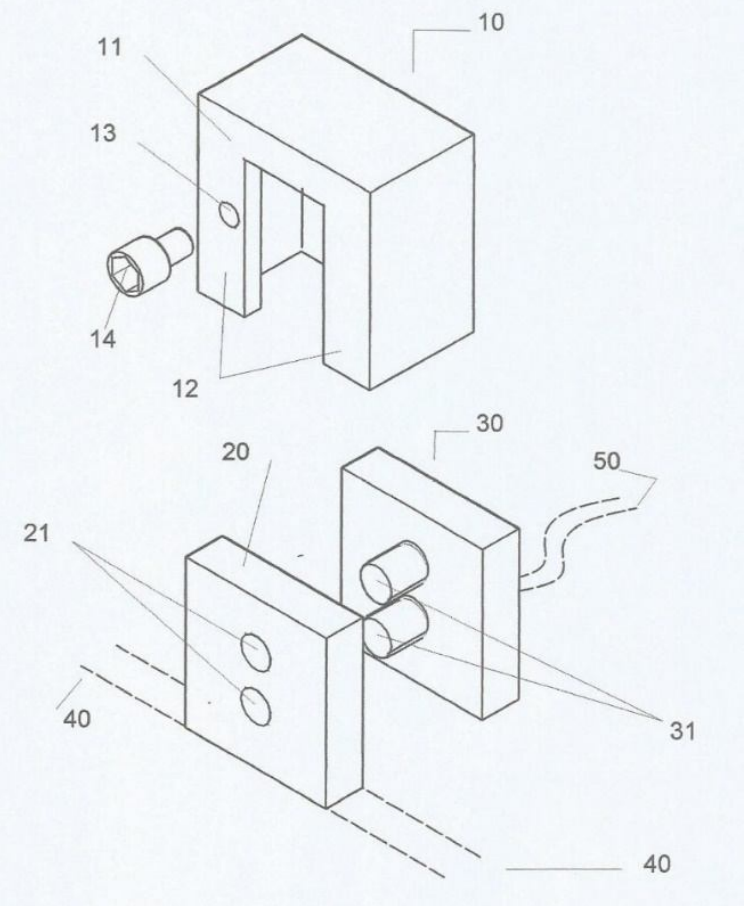

Figure 2: Dismantled View of the utility model, which shows the design and its parts 
The connector assembly 10 is made of a top plate 11 , a pair of elongated c-channel 12 attached to both ends of the said top plate. A threaded locking bore 13 is provided on one side of said channel 12. A fastening means 14 , preferably a bolt, is screwably mounted to the said threaded locking bore 13. The said connector assembly 10 is to be slidably mounted to said post 20 and support bar 30, respectively.

\subsection{Methodology}

This research employed the developmental research design(Jeff Galapon Pereyras, 2020) [5]. Adopted from (Jeff G Pereyras, 2019)[6] to determine the strength and advantages of the mounting device, the said mounting device has been demonstrated to the experts from the Mechanical Technology Department of the College of Technology, Pangasinan State University (PSU) Lingayen Campus, particularly its faculty members, and to the veteran tricycle users. Veteran tricycle users are those people handling a tricycle with a minimum of twenty (20) year-driving experience. During the demonstrations, interviews and consultation were facilitated to the same group of respondents to determine how effective the fabricated mounting device in terms of strength and advantages during the assembly and dismantling process of a motorcycle to its sidecar.

\section{RESULTS AND DISCUSSION}

This developmental study was narrowed only to the design and fabrication of mounting devices for connecting a motorcycle and a sidecar particularly in the assembly and dismantling process of a motorcycle to its sidecar. After the demonstration, interview and consultation with the experts from the College of Technology, Pangasinan State University - Lingayen Campus and to the veteran tricycle users, it was found that the said utility model was forceful and advantageous to its users particularly on the assembly and dismantling process of a motorcycle to its sidecar.

Having been tested for its strength and durability through a series of demonstrations, the authors decided to submit the said utility model for registration at the Intellectual Property Office of the Philippines. Last October and December 2019, the Industrial Design Certificate and Utility Model Certificate respectively were awarded by the same office to the said research output.

\section{CONCLUSION}

The fabricated mounting device for connecting a motorcycle to a sidecar, comprising; a post having a pair of bores mounted to a motorcycle chassis; a support bar having a pair of dowels attached to a reinforce bar of the said sidecar and fasten to the bores of the said post; a sleeve connector having an opposing side opening forming thereon an inside C-channel adapted to slide and interconnect said post and support bar; and a threaded locking bore provided on one side of said sleeve connector and fastening means screwably mounted to said threaded locking bore; wherein said means include a bolt threaded to said bore to secure the sleeve connector to said post and support bar.
Upon completion of the research, the tricycle users can now assemble and dismantle the motorcycle to its sidecar in seconds without sacrificing its strength and enjoying the advantages using the said utility model.

\section{RECOMMENDATIONS}

In this study, subsequently, the interview and consultation with the faculty members of the College of Technology, PSU Lingayen Campus particularly Mechanical Technology Department and the veteran tricycle users, the succeeding opinions were recommended; the device must have a provision for an interlocking system so that it can't be dismantled easily by the thieves thus taking motorcycle independently. Another, the utility model must have secretly installed screws with different head sizes so that the theft will need more than one (1) tool for dismantling purposes. Another, the utility model must have a connection switch series to the ignition system of the motorcycle as an anti-theft system of the whole tricycle. Lastly, the said utility model maybe subjected to the acceptability and technical feasibility testing [7] from the same group of respondents, and the maker and designer of the said research output may ask the assistance of a certain technology business incubation center for commercialization purposes [8].

\section{ACKNOWLEDGMENT}

Thank you very much to the Pangasinan State University Lingayen Campus.Thank you to Dr. Catalina Platon, the University Head for Intellectual Property and Research Utilization for the assistance in registering the utility model at the Intellectual Property Office of the Philippines. Also, a huge thanksand gratitude to Dr. Randy Joy M. Ventayen for mentoring and bearing a hand to the authors.

\section{REFERENCES}

[1] US4385770A - Motorcycle sidecar - Google Patents. (1983, May 31). Retrieved from:

https://patents.google.com/patent/US4385770A/en

[2] BASIC-SIDECAR-INSTRUCTIONS. (n.d.). Retrieved from http://www.dmcsidecars.com/wp-content/uploads/ 2017/02/BASIC-SIDECAR-INSTRUCTIONS.pdf

[3] 7 reasons taking a tricycle to work is so much better than driving a car. (2016, July 4). Retrieved from https://www.topgear.com.ph/features/feature-articles/7reasons-taking-a-tricycle-to-work-is-so-much-better-thandriving-a-car-a00024-20160704

[4] The Advantages and Disadvantages of Motorcycle and Tricycle in Nigeria. (2012, December 30). Retrieved from https://travel.wikinut.com/The-Advantages-andDisadvantages-of-Motorcycle-and-Tricycle-inNigeria/3v24xese/

[5] Pereyras, Jeff Galapon. (2020). Development of an electrical wiring installation trainer.International Journal of Scientific and Technology Research, 9(3), 4458-4461. https://doi.org/10.30534/ijatcse/2020/77932020

[6] Pereyras, Jeff G. (2019). Development of a Basic Electro-pneumatic Control Trainer.Asian Journal of Multidisciplinary Studies, 2(2). 
Engr. Juanito P. Maneclang Jr et al., International Journal of Emerging Trends in Engineering Research, 8(7), July 2020, 3645 - 3648

[7] Pereyras, J. G. (2020). Acceptability and technical feasibility of an electrical wiring installation trainer. International Journal of Advanced Trends in Computer Science and Engineering, 9(3), 2995-2999. DOI:10.30534/ijatcse/2020/77932020

[8] Esponilla II, F. D. (2019). Issues and challenges of technology business incubators in the Philippines. International Journal of Emerging Trends in Engineering Research, 7(9), 353-359.

DOI:10.30534/ijeter/2019/20792019 